\title{
Spin-magnetophonon level splitting in semimagnetic quantum wells
}

\author{
V. L. Gurevich and M. I. Muradov \\ A.F.Ioffe Institute, Russian Academy of Sciences, 194021 Saint Petersburg, Russia
}

\begin{abstract}
Spin-magnetophonon level splitting in a quantum well made of a semimagnetic wide gap semiconductor is considered. The semimagnetic semiconductors are characterized by a large effective $g$ factor. The resonance conditions $\hbar \omega_{L O}=\mu_{B} g B$ for the spin flip between two Zeeman levels due to interaction with longitudinal optical phonons can be achieved sweeping magnetic field $B$. This condition is studied in quantum wells. It is shown that it leads to a level splitting that is dependent on the electron-phonon coupling strength as well as on the spin-orbit interaction in this structure.

We treat in detail the Rashba model for the spin-orbit interaction assuming that the quantum well lacks inversion symmetry and briefly discuss other models. The resonant transmission and reflection of light by the well is suggested as a suitable experimental probe of the level splitting.
\end{abstract}

\section{INTRODUCTION}

The resonance coupling of Landau levels with longitudinal optical phonons (magnetophonon resonance) was theoretically predicted in [1] in the magnetoresistance investigation. The resonance takes place every time when the optical phonon frequency is the cyclotron frequency of an electron times some small integer. Thus a possibility has been pointed out for an internal resonance in solids. This phenomenon since its prediction has been observed in many experiments - see for instance the review [2].

A possibility of spin-flip transitions of electrons interacting with optical phonons between the Landau levels of opposite spin orientations that may be called spin-magnetophonon resonance (SMPR) - has been indicated and discussed in a number of papers (see $[3,4,45,6]$ ). The purpose of the present paper is to discuss the peculiarities of SMPR in semimagnetic semiconductors where due to large effective $g$-factors the corresponding interlevel spacing may be particularly large and therefore SMPR is well pronounced. The condition for the spin resonance has the form

$$
g \mu_{\mathrm{B}} B=\hbar \omega_{\mathrm{LO}} .
$$

Here $\mu_{\mathrm{B}}$ is the Bohr magneton, $g$ is the carrier effective $g$-factor while $B$ is the external magnetic field.

Many remarkable magnetooptical properties of wide gap semimagnetic semiconductors such as giant exchange splitting of the free exciton 7], giant Faraday effect [7, 8, 9], etc are determined by a large splitting of conduction and valence bands in magnetic field. This is a consequence of the exchange interaction of band carriers with the electrons of the half filled $d$ shell of the Mn ions. In the present paper we will treat as an example the compound $\mathrm{Cd}_{1-x} \mathrm{Mn}_{x} \mathrm{Te}_{\mathrm{e}}$ where the width of the gap between the top of valence band and the bottom of conduction band in the absence of magnetic field is given by $E_{g}=1.595+1.592 x \mathrm{eV}$.

In the presence of an external magnetic field the Mn ion spins are aligned along the magnetic field. Through the exchange of the Heisenberg type these spins interact with spins of the band carriers. Eventually, in the mean field model the band carrier dynamics can be described incorporating the exchange interaction only into the enhanced $g$-factor.

There are two competing mechanisms determining the sign and value of the exchange constant (and of the $g$ factor) [10, 11, 12]. The first mechanism originating from direct exchange interaction between the band and $d$ electrons is relatively weak and ferromagnetic. The second one is due to hybridization of $d$ orbitals and band states. The latter turns out to be antiferromagnetic and is negligible for the conduction band while for the valence band it determines the exchange constant.

The resonance coupling of Landau levels with optical phonons manifests itself also in a different way though the underlying physics is the same. It leads to magneto-optical anomalies both in bulk [13] and in two-dimensionally confined systems [14, 15]. Primary concern of Refs. 14, 15] was investigation of magneto-optical anomalies of optical phenomena in conventional GaAs based heterostructures. It was shown that magneto-optical anomalies in two dimensions provide a powerful tool for the electron-phonon coupling investigation in these structures. It was found that under the resonance condition with respect to electron-phonon interaction the relevant cyclotron peak splits into a doublet. This effect leads to anomalies in optical absorption and reflection (as well as in other optical effects such as for instance Raman scattering).

In the present paper we investigate this effect associated with SMPR, i. e. the magnetophonon resonances due to the spin flips. These electron-phonon resonance conditions can occur both for the valence and conduction electrons. The exchange constants for the conduction and valence electrons turn out to be different [16]. Though the resonance condition leading to the level splitting occurs first in the valence band we will show that the splitting itself is smaller for the valence band states than for the states in the conduction band.

In the next section we consider the level splitting as a formal quantum mechanical problem. This phenomenon can be understood in terms of degeneracy lifting of two degenerate states. The energy degeneracy of an electron 
state 2 and an electron in a state 1 plus an optical phonon (see Fig 1 ) is lifted by the electron-phonon interaction. We will obtain an expression for the level splitting not specifying the states involved in the relevant transitions. In Sec. III we determine the states and the energy levels of the conduction electrons taking into account the spinorbit interaction in the Rashba model. This allows to express the level splitting explicitly. We give the required estimations in the end of this section. As a possible experimental probe of this splitting phenomenon we propose the resonant reflection (transmission) of the light by a quantum well in the Faraday configuration. We consider the wave reflection (transmission) due to direct interband transitions, therefore in Sec. IV we give explicit expressions for the wave functions and energies of the valence band states. In section $\nabla$ these wave functions are used to determine the reflection and transmission coefficient of the light exciting interband transitions in the quantum well. In Sec. VI we discuss applicability of the perturabation theory for solution of our problem. We present conclusive remarks in Sec. VII.

\section{LEVEL SPLITTING}

We begin with treatment of a formal problem: we will consider two states 1 and 2 and find the self energy of an electron in the state 2 due to interaction of the electron with optical phonons. Suppose the energy of the state under consideration $\varepsilon_{2}$ is close to $\varepsilon_{1}+\hbar \omega_{\mathrm{LO}}$ (i.e. the electron state 2 and the electron state 1 plus the optical phonon with frequency $\omega_{\mathrm{LO}}$ are degenerate). This allows us to put aside all other possible electron states.

Generally, a single quantum well brings about new phonon (vibrational) modes. There could be three types of phonons associated with a quantum well [17]: phonons not penetrating into the quantum well, phonons peaking at the interface and decaying both in the well and in the barriers (interface phonons), and phonons confined to the well. The phonon Green function in the Matsubara technique can be written as

$$
D\left(\mathbf{r}_{\perp}, z, z^{\prime}, i \omega_{k}\right)=-\sum_{\alpha \mathbf{q}_{\perp}}\left|C_{\alpha}\right|^{2}\left(\frac{e^{i \mathbf{q}_{\perp} \mathbf{r}_{\perp}} \eta_{\alpha}(z) \eta_{\alpha}^{*}\left(z^{\prime}\right)}{i \omega_{k}+\hbar \omega_{\mathrm{LO}}}-\frac{e^{-i \mathbf{q}_{\perp} \mathbf{r}_{\perp}} \eta_{\alpha}^{*}(z) \eta_{\alpha}\left(z^{\prime}\right)}{i \omega_{k}-\hbar \omega_{\mathrm{LO}}}\right)
$$

where $\eta_{\alpha}(z)$ describes spatial distribution of the phonon $\alpha$ branch in the direction perpendicular to the well plane $(z$ axis), $\omega_{k}=2 \pi k T(k=0, \pm 1 \ldots)$ are the Matsubara boson frequencies, $\left|C_{\alpha}\right|^{2}$ is the electron-phonon coupling strength. $T$ is the temperature; we will use for it the energy units setting $k_{\mathrm{B}}=1$.

The electron-phonon interaction with longitudinal optical phonons can be treated in the bulk Fröhlich [18] model. According to the model $\eta_{\alpha}(z) \rightarrow e^{i q_{z} z},\left|C_{\alpha}\right|^{2} \rightarrow 2 \pi e^{2} \hbar \omega_{\mathrm{LO}} / q^{2} \epsilon^{*}$. This approximation in a relatively wide wells can be justified noting that the interaction with the interface phonons in this case can be neglected, interaction with the confined phonons qualitatively leads to the same result. Therefore, further on we will work in the Fröhlich approximation. We consider the dispersionless optical phonons, $\omega_{\text {LO }}$ being their frequency and

$$
\frac{1}{\epsilon^{*}}=\frac{1}{\epsilon_{\infty}}-\frac{1}{\epsilon_{0}}
$$

where $\epsilon_{\infty}\left(\epsilon_{0}\right)$ is the high frequency (static) limit of the dielectric susceptibility.

The electron self energy in the first approximation of the perturbation theory with respect to the electron-phonon interaction can be written as (see the diagram (a) in Fig.(7))

$$
\Sigma_{2}\left(i \varepsilon_{n}\right)=-T \frac{2 \pi \omega_{\mathrm{LO}} e^{2} \hbar}{\epsilon^{*}} \sum_{k} \frac{F_{21}}{i\left(\varepsilon_{n}-\omega_{k}\right)-\varepsilon_{1}+\mu} \frac{2 \hbar \omega_{\mathrm{LO}}}{\omega_{k}^{2}+\left(\hbar \omega_{\mathrm{LO}}\right)^{2}}
$$

where

$$
F_{21}=\int \frac{d^{3} q}{(2 \pi)^{3}} \frac{|<2| e^{i \mathbf{q r}}|1>|^{2}}{q^{2}}
$$

and

$$
\varepsilon_{n}=\pi(2 n+1) T
$$

To calculate the sum over $k$ in Eq.(4) we use

$$
G\left(i \varepsilon_{n}\right)=\frac{1}{\pi} \int_{-\infty}^{\infty} d \varepsilon \frac{\operatorname{Im} G^{R}(\varepsilon)}{\varepsilon-i \varepsilon_{n}}
$$

and

$$
D\left(i \omega_{k}\right)=\frac{1}{\pi} \int_{-\infty}^{\infty} d \varepsilon \frac{\operatorname{Im} D^{R}(\varepsilon)}{\varepsilon-i \omega_{k}}
$$


We define the phonon Green function $D^{R}$ as

$$
D^{R}(\omega)=\frac{1}{\omega-\hbar \omega_{\mathrm{LO}}+i 0}-\frac{1}{\omega+\hbar \omega_{\mathrm{LO}}+i 0} .
$$

The sum can be represented as

$$
T \sum_{k} G_{1}\left(i\left(\varepsilon_{n}-\omega_{k}\right)\right) D\left(i \omega_{k}\right)=\int_{-\infty}^{\infty} \frac{d x d y}{\pi^{2}} \frac{\operatorname{Im} G_{1}^{R}(x) \operatorname{Im} D^{R}(y)}{x+y-i \varepsilon_{n}}\left[1-n_{F}(x)+n_{B}(y)\right] .
$$

Here we have used the identities

$$
T \sum_{s} \frac{1}{i \varepsilon_{s}-x}=n_{F}(x)-1, T \sum_{k} \frac{1}{i \omega_{k}-y}=-\left[n_{B}(y)+1\right],
$$

where $n_{F}(x)\left[n_{B}(y)\right]$ is Fermi [Bose] function. The final result is

$$
\Sigma_{2}\left(i \varepsilon_{n}\right)=-\frac{2 \pi \omega_{\mathrm{LO}} e^{2} \hbar}{\epsilon^{*}} F_{21}\left\{\frac{n_{F}\left(\varepsilon_{1}\right)-n_{B}\left(\omega_{\mathrm{LO}}\right)-1}{i \varepsilon_{n}-\left(\varepsilon_{1}-\mu\right)-\hbar \omega_{\mathrm{LO}}}-\frac{n_{F}\left(\varepsilon_{1}\right)+n_{B}\left(\omega_{\mathrm{LO}}\right)}{i \varepsilon_{n}-\left(\varepsilon_{1}-\mu\right)+\hbar \omega_{\mathrm{LO}}}\right\} .
$$

Restricting ourselves with the low temperature case $T \ll \hbar \omega_{\text {LO }}$ and assuming that the state $\varepsilon_{1}$ is empty we get

$$
\Sigma_{2}\left(i \varepsilon_{n}\right)=\frac{\Delta^{2} / 4}{i \varepsilon_{n}-\left(\varepsilon_{1}-\mu\right)-\hbar \omega_{\mathrm{LO}}}
$$

where

$$
\Delta^{2}=\frac{8 \pi \omega_{\mathrm{LO}} e^{2} \hbar}{\epsilon^{*}} F_{21}
$$

For the electron Green function we get with this self energy

$$
G_{2}\left(i \varepsilon_{n}\right)=\frac{1}{i \varepsilon_{n}-\varepsilon_{2}+\mu-(\Delta / 2)^{2} /\left(i \varepsilon_{n}-\varepsilon_{1}-\hbar \omega_{L O}+\mu\right)} .
$$

We perform analytical continuation replacing $i \varepsilon_{n} \rightarrow \varepsilon+i 0$ and get for the retarded Green function

$$
G_{2}^{R}(\varepsilon)=\frac{\varepsilon-\varepsilon_{1}-\hbar \omega_{\mathrm{LO}}+\mu}{\left(\varepsilon-\varepsilon_{+}+\mu+i 0\right)\left(\varepsilon-\varepsilon_{-}+\mu+i 0\right)}
$$

where

$$
\varepsilon_{ \pm}=\frac{\varepsilon_{2}+\varepsilon_{1}+\hbar \omega_{\mathrm{LO}}}{2} \pm \sqrt{\left(\left(\varepsilon_{2}-\varepsilon_{1}-\hbar \omega_{\mathrm{LO}}\right) / 2\right)^{2}+(\Delta / 2)^{2}}
$$

As is seen from Eq. (16) we have gotten two poles of the Green function; the level $\varepsilon_{2}$ is split into a doublet with the energies $\varepsilon_{ \pm}$, the spacing between the poles being equal $\Delta$. The splitting can be expressed through the parameter $\alpha$ describing the effective mass polaron shift

$$
\Delta^{2}=16 \pi \alpha l_{\mathrm{LO}}\left(\hbar \omega_{\mathrm{LO}}\right)^{2} \int \frac{d \mathbf{q}}{(2 \pi)^{3}} \frac{|<2| e^{i \mathbf{q r}}|1>|^{2}}{q^{2}}, \alpha^{2}=\frac{m_{c} e^{4} / 2\left(\hbar \epsilon^{*}\right)^{2}}{\hbar \omega_{\mathrm{LO}}} .
$$

Here we introduced the length $l_{\mathrm{LO}}=\sqrt{\hbar / 2 m_{c} \omega_{\mathrm{LO}}}, m_{c}$ is the electron effective mass. The parameter $\alpha$ for materials with a relatively weak polarity is small. For instance, $\alpha=0.39$ for CdTe with partly ionic bonding. Suppose now that we can achieve the resonant condition $\varepsilon_{2}-\varepsilon_{1}=\hbar \omega_{\text {LO }}$ changing the interlevel spacing $\varepsilon_{2}-\varepsilon_{1}$. If the states 2 and 1 are the spin up and spin down states the resonant condition can be reached by adjusting external magnetic field. Since the level splitting is proportional to a matrix element $<2\left|e^{i \mathbf{q r}}\right| 1>$ we see that the phonons can lead to spin flips only provided the states 2 and 1 are not the eigenfunctions of spin operators $\mathbf{s}^{2}$ and $s_{z}$. For this reason, we must include into the Hamiltonian the spin-orbit interaction. We consider the spin-orbit interaction in the Rashba model [19]

$$
H_{R}=\frac{\alpha_{R}}{\hbar}[\boldsymbol{\sigma} \mathbf{p}] \mathbf{n}
$$

Here $\mathbf{n}$ is a unit vector perpendicular to the quantum well plane. This interaction is due to the structure inversion asymmetry. Parameter $\alpha_{R}$ is of the order of $10^{-9} \mathrm{eV} \cdot \mathrm{cm}$.

There could be another spin-orbit interaction term that is due to the bulk inversion asymmetry. The corresponding 3D spin-orbit Dresselhaus Hamiltonian [20] in the crystal principal axes reads

$$
H_{D}=\delta(\boldsymbol{\sigma} \mathbf{P})
$$




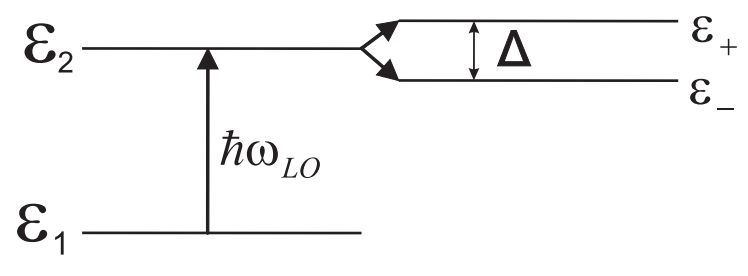

FIG. 1: Level splitting

Here $\hbar^{3} P_{x}=p_{y} p_{x} p_{y}-p_{z} p_{x} p_{z}$ and other components of $\mathbf{P}$ can be obtained by cyclic permutations. In $2 \mathrm{D}$ case this Hamiltonian takes the form (we omit the terms cubic in $p$ )

$$
H_{D}=\frac{\alpha_{D}}{\hbar}\left(\sigma_{y} p_{y}-\sigma_{x} p_{x}\right)
$$

where $\alpha_{D}=\delta<p_{z}^{2}>/ \hbar^{2}$ and $<p_{z}^{2}>$ is averaged over the transverse motion of the electron. The parameter $\alpha_{D}$ can be estimated as $10^{-10} \mathrm{eV} \cdot \mathrm{cm}$.

Being interested only in the possibility of the line splitting in the optical reflection (transmission) experiments with quantum wells explicit calculations for the spin-orbit interaction of the Rashba form will be presented since in many semiconductor nanostructures the Rashba interaction is stronger than the Dresselhaus one. However, it can be shown that the Dresselhaus term in the form (21) does not essentially differ from the Rashba term, so that to take into account the Dresselhaus term one should simply replace the constant $\alpha_{R}$ by $\alpha_{D}$ (this will be sufficient for estimations). Indeed, one can show that the Dresselhaus term can be obtained from the last term in Eq. (30) below simply replacing $\alpha_{R}$ by $\alpha_{D}$ and $a$ by $-i a$.

\section{DEEP QUANTUM WELL IN TRANSVERSE MAGNETIC FIELD}

Let $x, y$ be parallel to the quantum well plane, $z$ axis being perpendicular to the plane of the well. Further on we will consider the simplest case of an infinitely deep well. We assume that magnetic field $\mathbf{B}$ is along $z$ axis (perpendicular to the plane of the well) and choose the gauge $\mathbf{A}=B(0, x, 0)$. In wide gap semiconductors the conduction and valence bands can be considered separately. In the zinc blende structures the conduction band Hamiltonian near the point $\Gamma_{6}$ is

$$
\begin{gathered}
H=H_{0}+H_{R}, \\
H_{0}=\frac{\hbar^{2}}{2 m_{c}}\left(-i \nabla+\frac{e}{\hbar c} \mathbf{A}\right)^{2}+U+H_{Z} .
\end{gathered}
$$

Here we use the basis of $S s_{ \pm}$(where $S$ is the $S$-type Bloch amplitude and $s_{-}, s_{+}$are the two spin functions); $U$ is the confining potential of the quantum well. We write the Zeeman Hamiltonian as

$$
H_{Z}=\frac{1}{2} \mu_{B} \sigma_{z} g_{c} B
$$

Intending to consider semimagnetic semiconductors $\mathrm{Cd}_{1-x} \mathrm{Mn}_{x} \mathrm{Te}$ we will incorporate into the Hamiltonian the exchange Heisenberg interaction of the conduction band electrons with Mn ions

$$
H_{\mathrm{ce}}=-\sum_{n} J_{\mathrm{ce}}\left(\mathbf{r}-\mathbf{R}_{n}\right) \mathbf{S}_{n}^{\mathrm{Mn}} \mathbf{s}
$$

where $J_{\text {ce }}\left(\mathbf{r}-\mathbf{R}_{n}\right)$ is the exchange integral of the electron with the Mn ion localized at $\mathbf{R}_{n}$ site, the sum runs over all the Mn ions. We will use the mean-field approximation inserting the mean value of Mn spin in $z$ direction $\left\langle S_{z}^{\mathrm{Mn}}>\right.$ instead of the corresponding operator and ascribing spin $x<S_{z}^{\mathrm{Mn}}>$ to every crystal site. In this approximation the exchange Hamiltonian can be rewritten in the form

$$
H_{\mathrm{ce}}=-x<S_{z}^{\mathrm{Mn}}>N_{0}<S\left|J_{\mathrm{ce}}(\mathbf{r})\right| S>s_{z} \equiv-2 \hbar \omega_{c} V_{c} s_{z},
$$

where $N_{0}$ is the density of unit cells and $\left\langle S\left|J_{\text {ce }}(\mathbf{r})\right| S>\right.$ is the exchange integral (that is assumed to be positive). Here for convenience we factor out the cyclotron frequency $\omega_{c}=e B / m_{c} c$. The introduced quantity $V_{c}$ for the conduction band turns out to be negative and rather large. It can be written as

$$
V_{c}=x<S_{z}^{\mathrm{Mn}}>\frac{N_{0}<S\left|J_{\mathrm{ce}}(\mathbf{r})\right| S>}{2 \hbar \omega_{c}} .
$$


The induced Mn ion spin can be written as

$$
<S_{z}^{\mathrm{Mn}}>=-\mathcal{B}_{S}(\zeta), \quad \zeta=\frac{g_{\mathrm{Mn}} \mu_{B} B}{k_{B} T}
$$

where $\mathcal{B}_{S}(\zeta)$ is the Brillouin function

$$
\mathcal{B}_{S}(\zeta)=\frac{2 S+1}{2} \operatorname{coth}\left(\frac{2 S+1}{2} \zeta\right)-\frac{1}{2} \operatorname{coth}\left(\frac{\zeta}{2}\right) .
$$

For $S=5 / 2$

$$
\mathcal{B}_{5 / 2}(\zeta)=\frac{35}{12} \zeta, \quad \zeta \ll 1 ; \quad \mathcal{B}_{5 / 2}(\zeta)=\frac{5}{2}, \quad \zeta \gg 1,
$$

$g_{\mathrm{Mn}}=2, \mu_{B}$ is the Bohr magneton, $S=5 / 2$ is the spin of a manganese atom. Therefore, we see that $g_{c}$ in Eq. (24) must be understood as $g_{z z}-4 V_{c}$. Since $N_{0}<S\left|J_{c e}(\mathbf{r})\right| S>=0.22 \mathrm{eV}$ and $\hbar \omega_{c} \sim 1 \mathrm{meV}$ we get that $g_{c} \sim 50$.

Eigenfunctions of $H_{0}$ as functions of $y$ can be chosen as plane waves $e^{i k_{y} y} / \sqrt{L_{y}}$. As functions of $z$ they are the eigenfunctions $\chi_{i}(z)$ of an infinitely deep one-dimensional well with associated eigenvalues $\varepsilon_{i}$. Thus one can rewrite $H_{0}$ as

$$
H_{0}=\varepsilon_{i}-\frac{\hbar^{2}}{2 m_{c}} \frac{\partial^{2}}{\partial x^{2}}+\frac{m_{c} \omega_{c}^{2}}{2}\left(x-x_{0}\right)^{2}+H_{Z}
$$

Here the position of the center of oscillator $x_{0}=-k_{y} \hbar c / e B$ depends on the quasimomentum along $y$ direction $\hbar k_{y}$ (the motion along $y$ axis is free). The Rashba Hamiltonian in magnetic field is

$$
H_{R}=\alpha_{R}\left(\begin{array}{cc}
0 & \partial / \partial x+k_{y}+x / l_{c}^{2} \\
-\partial / \partial x+k_{y}+x / l_{c}^{2} & 0
\end{array}\right) .
$$

Here we have introduced the magnetic length $l_{c}=\sqrt{c \hbar / e B}$. Introducing Bose operators according to $\partial / \partial x=$ $\left(a-a^{\dagger}\right) /\left(\sqrt{2} l_{c}\right), x-x_{0}=l_{c}\left(a+a^{\dagger}\right) /(\sqrt{2})$ we get

$$
H=\varepsilon_{i}+\hbar \omega_{c}\left(a^{\dagger} a+1 / 2\right)+H_{Z}+\frac{\sqrt{2} \alpha_{R}}{l_{c}}\left(\begin{array}{cc}
0 & a \\
a^{\dagger} & 0
\end{array}\right) .
$$

The Rashba term does not change the ground state $\varphi_{0}\left(x-x_{0}\right)$ and its energy is $\varepsilon_{0}=\varepsilon_{i}+\hbar \omega_{c} / 2-\mu_{B} B g_{c} / 2$. Other eigenfunctions of $H$ are

$$
\psi_{n+}=\left(\begin{array}{c}
\cos u_{n} \varphi_{n}\left(x-x_{0}\right) \\
\sin u_{n} \varphi_{n+1}\left(x-x_{0}\right)
\end{array}\right)
$$

with eigenvalues

$$
\varepsilon_{n+}=\varepsilon_{i}+\hbar \omega_{c}(n+1)+\sqrt{\left(\frac{\hbar \omega_{c}-\mu_{B} g_{c} B}{2}\right)^{2}+2 \frac{\alpha_{R}^{2}}{l_{c}^{2}}(n+1)}
$$

and

$$
\psi_{n-}=\left(\begin{array}{c}
-\sin u_{n} \varphi_{n}\left(x-x_{0}\right) \\
\cos u_{n} \varphi_{n+1}\left(x-x_{0}\right)
\end{array}\right)
$$

with eigenvalues

$$
\varepsilon_{n-}=\varepsilon_{i}+\hbar \omega_{c}(n+1)-\sqrt{\left(\frac{\hbar \omega_{c}-\mu_{B} g_{c} B}{2}\right)^{2}+2 \frac{\alpha_{R}^{2}}{l_{c}^{2}}(n+1)},
$$

where

$$
\tan 2 u_{n}=2 \sqrt{2} \frac{\alpha_{R}}{l_{c}} \frac{\sqrt{n+1}}{\mu_{B} g_{c} B-\hbar \omega_{c}} .
$$

Here $\varphi_{n}$ are the oscillator functions of $x-x_{0}$

$$
\varphi_{n}\left(x-x_{0}\right)=\frac{1}{\pi^{1 / 4}} \frac{1}{\sqrt{2^{n} n !}} \frac{1}{l_{c}^{1 / 2}} \exp \left[-\left(x-x_{0}\right)^{2} / 2 l_{c}^{2}\right] H_{n}\left[\left(x-x_{0}\right) / l_{c}\right]
$$


where $H_{n}(x)$ are the Hermite polynomials. Thus we arrive at two groups of levels separated by (large) energy $\mu_{B} g_{c} B$. Both groups consist of sublevels that are nearly equidistant (if one neglects the spin-orbit contribution to the energy) separated by the cyclotron energy $\hbar \omega_{c}$. The minimal energy in the first group is $\varepsilon_{0}$, in the second group it is $\varepsilon_{0+}=\varepsilon_{0}+\mu_{B} g_{c} B$.

In what follows we restrict ourselves with the phonon induced transitions $0 \rightarrow 0+$ and $n-\rightarrow(n+1)+$. As we will see below under the realistic conditions the estimations show that the level splitting due to electron-phonon interaction turns out to be small as compared to the cyclotron energy, therefore it is sufficient to consider each pair of states separately. Indeed, for typical magnetic fields of the order of several tesla the magnetic length $l_{c}=\sqrt{\hbar c / e B} \sim 10$ $\mathrm{nm}$, and the cyclotron energy $\hbar \omega_{c}=\hbar^{2} / m_{c} l_{c}^{2} \sim 10^{-2} \mathrm{eV}$ while the level splitting is of the order $\hbar \Delta \omega \sim 10^{-3} \mathrm{eV}$ (see the estimations at the end of this section). Let us now calculate the matrix element between the ground state 0 of the first group and the $0+$ state of the second one

$$
\left|<i, 0+, k_{y}\right| e^{i q_{x} x+i q_{z} z}\left|i, 0, k_{y}-q_{y}>\right|^{2}=\sin ^{2} u_{0}|<i| e^{i q_{z} z}|i>|^{2} \frac{l_{c}^{2} q_{\perp}^{2}}{2} \exp \left(-\frac{l_{c}^{2} q_{\perp}^{2}}{2}\right) .
$$

or taking into account that $u_{0} \ll 1$

$$
\Delta_{0+, 0}^{2}=4 \alpha \frac{l_{\mathrm{LO}}}{l_{c}}\left(\hbar \omega_{\mathrm{LO}}\right)^{2} u_{0}^{2} f\left(\frac{l_{c}}{L}\right)
$$

where

$$
f\left(\frac{l_{c}}{L}\right)=l_{c} \int d z_{1} d z_{2} \chi_{i}^{2}\left(z_{1}\right) \chi_{i}^{2}\left(z_{2}\right) \int_{0}^{\infty} d q_{\perp} \frac{q_{\perp}^{2} l_{c}^{2}}{2} \exp \left(-l_{c}^{2} q_{\perp}^{2} / 2-q_{\perp}\left|z_{1}-z_{2}\right|\right) .
$$

Transitions from $n-$ to $(n+1)+$ states are resonant too; for these transitions we have (omitting $k_{y}$ and $\left.k_{y}-q_{y}\right)$

$$
\begin{array}{r}
|<i,(n+1)+| e^{i q_{x} x+i q_{z} z}|i, n->|^{2}=2\left(\frac{\alpha_{R}}{l_{c} \mu_{B} g_{c} B}\right)^{2}|<i| e^{i q_{z} z}|i>|^{2} \\
\times \frac{l_{c}^{2} q_{\perp}^{2}}{2} e^{-l_{c}^{2} q_{\perp}^{2} / 2}\left[L_{n+1}^{1}\left(l_{c}^{2} q_{\perp}^{2} / 2\right)-L_{n}^{1}\left(l_{c}^{2} q_{\perp}^{2} / 2\right)\right]^{2}
\end{array}
$$

where $L_{n}^{\alpha}(x)$ are the Laguerre polynomials defined as in Ref.[21]. Here we have taken into account that $\sin u_{n} \simeq u_{n} \simeq$ $\sqrt{2(n+1)} \alpha_{R} / l_{c} \mu_{B} B$. Since $L_{n+1}^{\alpha-1}(x)=L_{n+1}^{\alpha}(x)-L_{n}^{\alpha}(x)$ this expression can be simplified

$$
|<i,(n+1)+| e^{i q_{x} x+i q_{z} z}|i, n->|^{2}=2\left(\frac{\alpha_{R}}{l_{c} \mu_{B} g_{c} B}\right)^{2}|<i| e^{i q_{z} z}|i>|^{2} \frac{l_{c}^{2} q_{\perp}^{2}}{2} e^{-l_{c}^{2} q_{\perp}^{2} / 2}\left[L_{n+1}\left(l_{c}^{2} q_{\perp}^{2} / 2\right)\right]^{2} .
$$

We have

$$
\begin{gathered}
\Delta_{(n+1)+, n-}^{2}=8 \alpha \frac{l_{\mathrm{LO}}}{l_{c}}\left(\hbar \omega_{\mathrm{LO}}\right)^{2}\left(\frac{\alpha_{R}}{l_{c} \mu_{B} g_{c} B}\right)^{2} f_{n}\left(\frac{l_{c}}{L}\right), \\
f_{n}\left(\frac{l_{c}}{L}\right)=l_{c} \int d z_{1} d z_{2} \chi_{i}^{2}\left(z_{1}\right) \chi_{i}^{2}\left(z_{2}\right) \int_{0}^{\infty} d q_{\perp} \frac{l_{c}^{2} q_{\perp}^{2}}{2}\left[L_{n+1}\left(\frac{l_{c}^{2} q_{\perp}^{2}}{2}\right)\right]^{2} e^{-l_{c}^{2} q_{\perp}^{2} / 2-q_{\perp}\left|z_{1}-z_{2}\right|}
\end{gathered}
$$

Here we give an estimation for $\Delta_{0+, 0}^{2}$ assuming that the transverse motion is described by the wave function $\chi_{1}(z)=$ $\sqrt{2 / L} \sin (\pi z / L)[$ see Eq.(37)]

$$
\Delta_{0+, 0}^{2}=8 \alpha\left(\hbar \omega_{\mathrm{LO}}\right)^{2}\left(\frac{\alpha_{R}}{l_{c} \mu_{B} g_{c} B}\right)^{2} \frac{l_{\mathrm{LO}}}{l_{c}} f\left(\frac{l_{c}}{L}\right),
$$

where $f(x)=\sqrt{2 \pi} / 4$ for $x \gg 1$ and $f(x)=3 x / 2$ for $x \ll 1$ (see Fig 2). Taking into account the CdTe parameters, namely the longitudinal optical phonon frequency $\omega_{\mathrm{LO}}=3.22 \cdot 10^{13} \mathrm{~s}^{-1}(246 \mathrm{~K})$, the susceptibilities $\epsilon_{0}=10.3$ and $\epsilon_{\infty}=6.9$, the effective electron mass $m_{c}=0.1 m_{0}\left(m_{0}\right.$ is the free electron mass $)$ we see that the line splitting is $\Delta \omega=\Delta / \hbar \simeq \alpha_{R} \sqrt{\alpha} / \hbar l_{c} \sim 10^{11} \mathrm{~s}^{-1}$. For the Dresselhaus interaction the line splitting would be $\alpha_{D} / \alpha_{R}$ times smaller.

\section{VALENCE BAND}

In the structures having zinc blende symmetry the valence $\Gamma_{8}$ band is described by the Luttinger Hamiltonian

$$
H=H_{0}+H\left(k_{z}\right),
$$




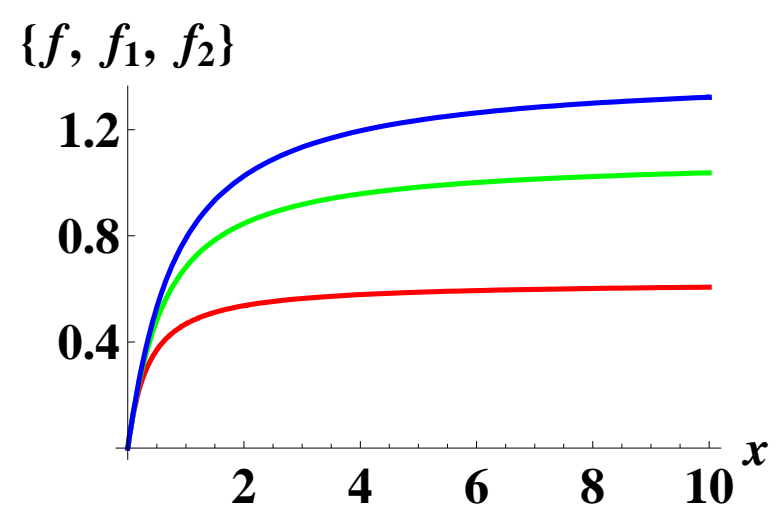

FIG. 2: Functions $f(x), f_{0}(x)$ and $f_{1}(x)$. The second function corresponds to $\Delta_{1+, 0-}^{2}$ and saturates at $x \gg 1$ reaching the value $7 \sqrt{2 \pi} / 16$ while the third one corresponds to the transition $1-\rightarrow 2+$ and also saturates at $x \gg 1$ reaching the value $145 \sqrt{2 \pi} / 256$

where we separate the part $H\left(k_{z}\right)$ depending on $k_{z}$,

$$
\begin{gathered}
H_{0}=-\frac{\hbar^{2}}{2 m_{0}}\left\{\left(\gamma_{1}+\frac{5}{2} \gamma_{2}\right)\left(k_{x}^{2}+k_{y}^{2}\right)-2 \gamma_{2}\left(J_{x}^{2} k_{x}^{2}+J_{y}^{2} k_{y}^{2}\right)-4 \gamma_{3}\left\{J_{x} J_{y}\right\}\left\{k_{x} k_{y}\right\}+2 \frac{e}{c} \kappa \mathbf{J B}\right\}, \\
H\left(k_{z}\right)=-\frac{\hbar^{2}}{2 m_{0}}\left\{\left(\gamma_{1}+\frac{5}{2} \gamma_{2}\right) k_{z}^{2}-2 \gamma_{2} J_{z}^{2} k_{z}^{2}-4 \gamma_{3}\left(\left\{J_{x} J_{z}\right\}\left\{k_{x} k_{z}\right\}+\left\{J_{y} J_{z}\right\}\left\{k_{y} k_{z}\right\}\right)\right\} .
\end{gathered}
$$

Here $\gamma_{1}, \gamma_{2}, \gamma_{3}, \kappa$ are material parameters, $\mathbf{J}$ is the operator of angular momentum $J=3 / 2$, the symmetrized products are defined according to

$$
\{A B\}=\frac{A B+B A}{2} .
$$

We add to the valence band Hamiltonian the exchange Heisenberg interaction of the valence band electrons with Mn ions

$$
H_{v e}=-\sum_{n} J_{v e}\left(\mathbf{r}-\mathbf{R}_{n}\right) \mathbf{S}_{n}^{\mathrm{Mn}} \mathbf{s}
$$

where $J_{v e}\left(\mathbf{r}-\mathbf{R}_{n}\right)$ is the exchange integral of a valence band electron with a $\mathrm{Mn}$ ion.

The wave function can be written as

$$
\Psi=\sum_{i} F_{i}(\mathbf{r}) u_{i}(\mathbf{r})
$$

where $u_{i}(\mathbf{r})$ are the four degenerate states at the top of the valence band [22]

$$
u_{ \pm 3 / 2}=\mp \frac{1}{\sqrt{2}}(X \pm i Y) s_{ \pm}, \quad u_{ \pm 1 / 2}=\frac{1}{\sqrt{3}}\left[\mp \frac{1}{\sqrt{2}}(X \pm i Y) s_{\mp}+\sqrt{2} Z s_{ \pm}\right]
$$

It is easily seen that in this basis the spin operator $s_{z}=\sigma_{z} / 2$ is also diagonal and is related to the $J_{z}$ operator by $s_{z}=J_{z} / 3$, therefore we can rewrite the exchange Hamiltonian as

$$
H_{e}=-x<S_{z}^{\mathrm{Mn}}>N_{0}<X\left|J_{v e}(\mathbf{r})\right| X>\frac{1}{3} J_{z} \equiv-2 \hbar \omega_{c 0} V_{v} J_{z}
$$

Here for convenience we factor out the cyclotron frequency $\omega_{c 0}=e B / m_{0} c$ anticipating its appearance in the following formulae. The introduced quantity $V_{v}$ for the valence band turns out to be positive and rather large. It can be estimated as

$$
V_{v}=x<S_{z}^{\mathrm{Mn}}>\frac{N_{0}<X\left|J_{v e}(\mathbf{r})\right| X>}{6 \hbar \omega_{c 0}} .
$$

Here the exchange integral for the valence band $\left\langle X\left|J_{v e}(\mathbf{r})\right| X\right\rangle$ is negative. 
At the typical magnetic fields of the order of several tesla the magnetic length $l_{c}=\sqrt{\hbar c / e B} \sim 10 \mathrm{~nm}$, and the cyclotron energy $\hbar \omega_{c 0}=\hbar^{2} / m_{0} l_{c}^{2} \sim 10^{-3} \mathrm{eV}$, while $N_{0}\left\langle X\left|J_{v e}(\mathbf{r})\right| X\right\rangle=-0.88 \mathrm{eV}$. Therefore $V_{v} \gg 1$.

We again choose the gauge $\mathbf{A}=B(0, x, 0)$ and introduce the operators $a, a^{\dagger}$ according to

$$
k_{x}=-\frac{i}{\sqrt{2}_{c}}\left(a-a^{\dagger}\right), k_{y}=\frac{1}{\sqrt{2} l_{c}}\left(a+a^{\dagger}\right) .
$$

Replacing also the operators $J_{x}, J_{y}$ by $J_{ \pm}=J_{x} \pm i J_{y}$ we get

$$
\begin{gathered}
H_{0}=-\hbar \omega_{c 0}\left\{\left[\gamma_{1}-\frac{5}{4} \gamma_{2}+\gamma_{2} J_{z}^{2}\right]\left(a^{\dagger} a+1 / 2\right)+\frac{\gamma_{2}}{4}\left(J_{-}^{2}+J_{+}^{2}\right)\left[a^{2}+\left(a^{\dagger}\right)^{2}\right]+\frac{\gamma_{3}}{4}\left(J_{+}^{2}-J_{-}^{2}\right)\left[a^{2}-\left(a^{\dagger}\right)^{2}\right]+\frac{e}{c} l_{c}^{2} \kappa \mathbf{J B}\right\}, \\
H\left(k_{z}\right)=-\hbar \omega_{c 0}\left\{a J_{+}\left(J_{z}+\frac{1}{2}\right)-a^{\dagger} J_{-}\left(J_{z}-1 / 2\right)\right\} i \sqrt{2} \gamma_{3}\left(l_{c} k_{z}\right)-\hbar \omega_{c 0} \frac{1}{2}\left\{\gamma_{1}+\frac{5}{2} \gamma_{2}-2 \gamma_{2} J_{z}^{2}\right\}\left(l_{c} k_{z}\right)^{2} .
\end{gathered}
$$

Further on we will use the spherical approximation, i.e. we set $\gamma_{2}=\gamma_{3}$. We get

$$
\begin{aligned}
-\frac{H}{\hbar \omega_{c 0}}= & 2 g_{v} J_{z}+\left(\gamma_{1}-\frac{5}{4} \gamma_{2}+\gamma_{2} J_{z}^{2}\right)\left(a^{\dagger} a+1 / 2\right)+\frac{1}{2}\left\{\gamma_{1}+\frac{5}{2} \gamma_{2}-2 \gamma_{2} J_{z}^{2}\right\}\left(l_{c} k_{z}\right)^{2} \\
& +\frac{\gamma_{2}}{2}\left[J_{+}^{2} a^{2}+J_{-}^{2}\left(a^{\dagger}\right)^{2}\right]+\left\{a J_{+}\left(J_{z}+1 / 2\right)-a^{\dagger} J_{-}\left(J_{z}-1 / 2\right)\right\} i \sqrt{2} \gamma_{2}\left(l_{c} k_{z}\right)
\end{aligned}
$$

where we have introduced the effective $g$ factor in the valence band $g_{v}=\hbar \kappa / 2+V_{v}$.

Due to large values of the exchange Hamiltonian $g_{v}$ we can omit the last two terms, i.e.

$$
V=\left\{a J_{+}\left(J_{z}+1 / 2\right)-a^{\dagger} J_{-}\left(J_{z}-1 / 2\right)\right\} \sqrt{2} \gamma_{2} l_{c} \frac{\partial}{\partial z}+\frac{\gamma_{2}}{2}\left(J_{+}^{2} a^{2}+J_{-}^{2}\left(a^{\dagger}\right)^{2}\right) .
$$

in Eq. (53) that sufficiently simplifies the problem. The reason of such a separation of the Hamiltonian is rather obvious, the Hamiltonian $V$ leads to transitions changing both spin and Landau numbers and can be taken into account as a perturbation. In this approximation the levels can be considered independently and we have for the top heavy and light hole series of levels (in the hole representation)

$$
\begin{gathered}
E_{-3 / 2, n, n_{v}}^{(\mathrm{hh})}=E_{g}-3 \hbar \omega_{c 0} g_{v}+\hbar \omega_{c 0} \frac{m_{0}\left(3 m_{h}+m_{l}\right)}{4 m_{l} m_{h}}(n+1 / 2)+\frac{\pi^{2} \hbar^{2} n_{v}^{2}}{2 m_{h} L^{2}} \\
\psi_{-3 / 2}^{(\mathrm{hh})}=\varphi_{n}\left(x-x_{0 k_{y}}\right) \chi_{n_{v}}(z) \frac{e^{i k_{y} y}}{\sqrt{L_{y}}} u_{-3 / 2} \\
E_{-1 / 2, n, n_{v}}^{(\mathrm{lh})}=E_{g}-\hbar \omega_{c 0} g_{v}+\hbar \omega_{c 0} \frac{m_{0}\left(3 m_{l}+m_{h}\right)}{4 m_{l} m_{h}}(n+1 / 2)+\frac{\pi^{2} \hbar^{2} n_{v}^{2}}{2 m_{l} L^{2}} \\
\psi_{-1 / 2}^{(\mathrm{lh})}=\varphi_{n}\left(x-x_{0 k_{y}}\right) \chi_{n_{v}}(z) \frac{e^{i k_{y} y}}{\sqrt{L_{y}}} u_{-1 / 2}
\end{gathered}
$$

Here $E_{g}$ is the gap, $m_{l}\left(m_{h}\right)$ are the light (heavy) hole masses, $n_{v}$ is the quantization number of transverse motion and $\chi_{n_{v}}(z)$ is the corresponding wave function. We take into account that the $\gamma_{1}, \gamma_{2}$ parameters are related to effective masses by $\gamma_{1}=m_{0}\left(m_{h}+m_{l}\right) / 2 m_{h} m_{l}$ and $\gamma_{2}=m_{0}\left(m_{h}-m_{l}\right) / 4 m_{h} m_{l}$.

In this zeroth approximation phonons can not induce transitions between these states. In the next approximation of perturbation theory with respect to $V$ these states are mixed and we get for the top heavy hole state $\psi_{-3 / 2, n, n_{v}}^{(\mathrm{hh})}$

$$
\psi_{-3 / 2,0,1}^{(\mathrm{hh})}=\varphi_{0}\left(x-x_{0 k_{y}}\right) \chi_{1}(z) \frac{e^{i k_{y} y}}{\sqrt{L_{y}}} u_{-3 / 2} .
$$

For the light hole top state we have

$$
\psi_{-1 / 2,0,1}^{(\mathrm{lh})}=\frac{e^{i k_{y} y}}{\sqrt{L_{y}}}\left\{\varphi_{0}\left(x-x_{0 k_{y}}\right) \chi_{1}(z) u_{-1 / 2}+\frac{4}{3} \frac{\gamma_{2} \hbar \omega_{c 0}\left(l_{c} / L\right)}{E_{-1 / 2,0,1}-E_{-3 / 2,1,2}} \varphi_{1}\left(x-x_{0 k_{y}}\right) \chi_{2}(z) u_{-3 / 2}\right\} .
$$

Now it is obvious that phonon can induce transitions between these states. Suppose that sweeping the magnetic field we achieve the hole-phonon resonance condition between the states described by Eq.(59) and Eq.(60)

$$
E_{-1 / 2,0,1}-E_{-3 / 2,0,1}=\hbar \omega_{\mathrm{LO}}
$$


or

$$
2 g_{v} \hbar \omega_{c 0}-\frac{m_{0}\left(m_{h}-m_{l}\right)}{4 m_{h} m_{l}}\left(\hbar \omega_{c 0}-2 \frac{\pi^{2} \hbar^{2}}{m_{0} L^{2}}\right)=\hbar \omega_{\mathrm{LO}}
$$

For the value $\Delta_{-1 / 2,-3 / 2} \equiv \Delta_{v}$ describing the splitting in the valence band we get at the resonant condition

$$
\Delta_{v}^{2}=4 \alpha \frac{l_{\mathrm{LO}}}{l_{c}}\left(\hbar \omega_{\mathrm{LO}}\right)^{2} 6\left(\frac{m_{0}\left(m_{h}-m_{l}\right)}{4 m_{h} m_{l}}\right)^{2}\left(\frac{\hbar \omega_{c 0}}{\hbar \omega_{\mathrm{LO}}}\right)^{2}\left(\frac{l_{c}}{L}\right)^{2} f_{v}\left(l_{c} / L\right),
$$

where

$$
f_{v}\left(\frac{l_{c}}{L}\right)=l_{c} \int d z_{1} d z_{2} \chi_{1}\left(z_{1}\right) \chi_{2}\left(z_{1}\right) \chi_{1}\left(z_{2}\right) \chi_{2}\left(z_{2}\right) \int_{0}^{\infty} d q_{\perp} \frac{q_{\perp}^{2} l_{c}^{2}}{2} \exp \left(-l_{c}^{2} q_{\perp}^{2} / 2-q_{\perp}\left|z_{1}-z_{2}\right|\right)
$$

$f_{v}(x)=x, x \ll 1, \quad f_{v}(x)=\left(10 / 9 \pi^{2} x\right), x \gg 1$.

Let us compare the SMPR splittings in the conduction and valence bands. We evaluate

$$
\frac{\Delta_{0+, 0}}{\Delta_{v}} \sim\left(\frac{\alpha_{R}}{L \hbar^{2} / 2 m_{0} L^{2}}\right)\left(\frac{g_{v}}{g_{c}}\right)^{3 / 4}\left(\frac{4 m_{h} m_{l}}{m_{0}\left(m_{h}-m_{l}\right)}\right)\left(\frac{f\left(l_{c} / L\right)}{f_{v}\left(2 \sqrt{g_{v} / g_{c}} l_{c} / L\right)}\right)^{1 / 2}
$$

and see that the splitting in the conduction band is bigger than in the valence band and is determined by the parameter $g_{v} / g_{c}$. Here $l_{c}$ is the magnetic length for magnetic fields required to achieve the resonance condition in the conduction band.

In principle, in valence band one can also write the spin-orbital term of Rashba type [23]

$$
H_{v R}=\frac{\alpha^{\prime}}{\hbar}[\mathbf{J} \mathbf{p}] \mathbf{n}
$$

that in magnetic field can be rewritten as

$$
H_{v R}=\frac{\alpha^{\prime}}{\sqrt{2} l_{c}}\left\{J_{+} a+J_{-} a^{+}\right\}
$$

This term leads to the ratio

$$
\frac{\Delta_{0+, 0}}{\Delta_{v}} \sim\left(\frac{\alpha_{R}}{\alpha^{\prime}}\right)\left(\frac{g_{v}}{g_{c}}\right)^{3 / 4}\left(\frac{f\left(l_{c} / L\right)}{f_{v}^{\prime}\left(2 \sqrt{g_{v} / g_{c}} l_{c} / L\right)}\right)^{1 / 2}
$$

where

$$
f_{v}^{\prime}\left(\frac{l_{c}}{L}\right)=\int d z_{1} d z_{2} \chi_{1}^{2}\left(z_{1}\right) \chi_{2}^{2}\left(z_{2}\right) \int_{0}^{\infty} d q q^{4} \exp \left(-q^{2} / 2-q\left|z_{1}-z_{2}\right| / l_{c}\right) .
$$

Although the SMPR condition are met first for the hole states as one sweeps the magnetic field the splitting in the valence band turns out to be much smaller than in the conduction band. This is the consequence of the smaller spin-phonon coupling strength for the states strongly shifted by the Zeeman energy.

\section{RESONANT REFLECTION AND TRANSMISSION}

We consider the simplest geometry where the wave $\sim e^{i k z}$ is incident perpendicularly to the plane of the well. Neglecting in the induced current the longitudinal part (this term in the induced current has a small factor $u_{0} \simeq$ $\left.\alpha_{R} / \mu_{B} g_{c} B l_{c}\right)$ so that we can put $\nabla \cdot \mathbf{D}=\epsilon_{b} \nabla \cdot \mathbf{E}=0$ the Maxwell equation for the wave with frequency $\omega$ can be written as (in this section $k$ denotes the wave vector of light)

$$
\frac{d^{2}}{d z^{2}} E_{\alpha}+k^{2} E_{\alpha}=\frac{4 \pi}{\hbar c^{2}} \int d z^{\prime} \Pi_{\alpha \beta}^{R}\left(z, z^{\prime}, \omega\right) E_{\beta}\left(z^{\prime}\right)
$$

Here $k^{2}=\omega^{2} \epsilon_{b} / c^{2}$ (we neglect the difference in the background susceptibilities of the well and barriers). We have taken into account that the polarization operator $\Pi_{\alpha \beta}$ (here the averaging over the distances much greater than the lattice parameter is implied) is

$$
\Pi^{R}\left(z, z^{\prime}, \omega\right)=\int d x^{\prime} d y^{\prime} \Pi^{R}\left(\mathbf{r}, \mathbf{r}^{\prime}, \omega\right)
$$


The Green function of operator $d^{2} / d z^{2}+k^{2}$ obeys the equation

$$
\left(\frac{d^{2}}{d z^{2}}+k^{2}\right) \mathcal{G}\left(z, z^{\prime}\right)=-\delta\left(z-z^{\prime}\right)
$$

and is given by

$$
\mathcal{G}^{ \pm}\left(z, z^{\prime}\right)= \pm \frac{i}{2 k} e^{ \pm i k\left|z-z^{\prime}\right|}
$$

For the transmission and reflection problem one should use $\mathcal{G}^{+}\left(z, z^{\prime}\right)$ function. Then the solution of Eq. (63) can be written as

$$
E_{\alpha}=E_{\alpha}^{0} e^{i k z}-\frac{4 \pi}{\hbar c^{2}} \int d z^{\prime} d z^{\prime \prime} \mathcal{G}^{+}\left(z, z^{\prime}\right) \Pi_{\alpha \beta}^{R}\left(z^{\prime}, z^{\prime \prime}, \omega\right) E_{\beta}\left(z^{\prime \prime}\right)
$$

where $E_{\alpha}^{0}$ is the amplitude of the incident wave. For $z>L$, where $L$ is the width of the quantum well, we can identify the transmitted wave as

$$
E_{\alpha}^{t}=E_{\alpha}^{0} e^{i k z}-\frac{2 i \pi}{k \hbar c^{2}} e^{i k z} \int_{0}^{L} d z^{\prime} d z^{\prime \prime} e^{-i k z^{\prime}} \Pi_{\alpha \beta}^{R}\left(z^{\prime}, z^{\prime \prime}, \omega\right) E_{\beta}\left(z^{\prime \prime}\right)
$$

and the reflected one can be identified considering $z<0$

$$
E_{\alpha}^{r}=-\frac{2 i \pi}{k \hbar c^{2}} e^{-i k z} \int_{0}^{L} d z^{\prime} d z^{\prime \prime} e^{i k z^{\prime}} \Pi_{\alpha \beta}^{R}\left(z^{\prime}, z^{\prime \prime}, \omega\right) E_{\beta}\left(z^{\prime \prime}\right)
$$

Assuming that $\Pi^{R}\left(z, z^{\prime}\right)$ can be factorized as $\Pi^{R}\left(z, z^{\prime}\right)=\Pi^{(1)}(z) \Pi^{(2)}\left(z^{\prime}\right)$ (such a factorization is possible since below we will consider transitions between two fixed states with respect to transverse motion $\left.\chi_{n_{v}}(z), \chi_{n_{c}}(z)\right)$ we scalarly multiply Eq. (67) by $\Pi_{\alpha}^{(2)}(z)$ and integrate over $z$, then we get

$$
\mathcal{F}=-\mathcal{F} \frac{4 \pi}{\hbar c^{2}} \int d z d z^{\prime} \mathcal{G}^{+}\left(z, z^{\prime}\right) \Pi_{\alpha \alpha}^{R}\left(z, z^{\prime}, \omega\right)+E_{\alpha}^{0} \int_{0}^{L} d z e^{i k z} \Pi_{\alpha}^{(2)}(z)
$$

where we have introduced notation

$$
\mathcal{F}=\int_{0}^{L} d z^{\prime} \Pi_{\beta}^{(2)}\left(z^{\prime}, \omega\right) E_{\beta}\left(z^{\prime}\right)
$$

Solving Eq. (70) for $\mathcal{F}$ and making use of Eq. (68) and Eq. (69) we get for the amplitudes of the transmitted and reflected waves

$$
\begin{gathered}
E_{\alpha}^{t}=\left(\delta_{\alpha \beta}+\frac{4 \pi \int d z^{\prime} d z e^{-i k\left(z-z^{\prime}\right)} \Pi_{\alpha \beta}^{R}\left(z, z^{\prime}, \omega\right)}{2 i k c^{2} \hbar-4 \pi \int d z^{\prime} d z e^{i k\left|z-z^{\prime}\right|} \Pi_{\gamma \gamma}^{R}\left(z, z^{\prime}, \omega\right)}\right) E_{\beta}^{0}, \\
E_{\alpha}^{r}=\frac{4 \pi \int d z^{\prime} d z e^{i k\left(z+z^{\prime}\right)} \Pi_{\alpha \beta}^{R}\left(z, z^{\prime}, \omega\right)}{2 i k c^{2} \hbar-4 \pi \int d z^{\prime} d z e^{i k\left|z-z^{\prime}\right| \Pi_{\gamma \gamma}^{R}\left(z, z^{\prime}, \omega\right)}} E_{\beta}^{0} .
\end{gathered}
$$

In the basis $\mathbf{e}_{ \pm}=\left(\mathbf{e}_{x} \pm i \mathbf{e}_{y}\right) / \sqrt{2}$ in our approximation only one component of $\Pi_{\alpha \beta}$ is nonvanishing, i.e. $\Pi_{++}=2 \Pi_{x x}$. Due to the symmetry relations we have $\Pi_{x x}=\Pi_{y y}=i \Pi_{x y}=-i \Pi_{y x}$. Therefore, left circularly polarized incident wave $\mathbf{e}_{-}$is not reflected, while for the right polarized incident wave $\mathbf{e}_{+}$we get

$$
t_{+}=1+\frac{4 \pi \int d z^{\prime} d z e^{-i k\left(z-z^{\prime}\right)} \Pi_{++}^{R}\left(z, z^{\prime}, \omega\right)}{2 i k c^{2} \hbar-4 \pi \int d z^{\prime} d z e^{i k\left|z-z^{\prime}\right|} \Pi_{++}^{R}\left(z, z^{\prime}, \omega\right)}
$$

for the transmission coefficient $\mathbf{E}^{t}=t_{+} E_{+}^{0} \mathbf{e}_{+} e^{i k z}$ and

$$
r_{+}=\frac{4 \pi \int d z^{\prime} d z e^{i k\left(z+z^{\prime}\right)} \Pi_{++}^{R}\left(z, z^{\prime}, \omega\right)}{2 i k c^{2} \hbar-4 \pi \int d z^{\prime} d z e^{i k\left|z-z^{\prime}\right|} \Pi_{++}^{R}\left(z, z^{\prime}, \omega\right)}
$$

for the reflection (amplitude) coefficient $\mathbf{E}^{r}=r_{+} E_{+}^{0} \mathbf{e}_{+} e^{-i k z}$. Since the propagation direction of the wave is now inverted the reflected wave has the left polarization. A linearly polarized incident wave will be reflected as a circularly 
left polarized wave. In the case where the wave length $2 \pi / k$ is bigger than the well width $L$ (i.e. $k L \ll 1$ ) the exponential factors can be omitted.

Let us consider the polarization operator. We can write the formal expression for the operator

$$
\begin{array}{r}
\Pi_{\alpha \beta}^{R}\left(\mathbf{r}, \mathbf{r}^{\prime}, \omega\right)=-\frac{i}{2} \sum_{\lambda_{1} \lambda_{2}} j_{\lambda_{2} \lambda_{1}}^{\alpha}(\mathbf{r}) j_{\lambda_{1} \lambda_{2}}^{\beta}\left(\mathbf{r}^{\prime}\right) \int \frac{d \varepsilon}{2 \pi \hbar}\left\{\tanh \frac{\varepsilon+\hbar \omega}{2 T}\left(G_{\lambda_{1}}^{R}(\varepsilon / \hbar+\omega)-G_{\lambda_{1}}^{A}(\varepsilon / \hbar+\omega)\right) G_{\lambda_{2}}^{A}(\varepsilon / \hbar)\right. \\
\left.+\tanh \frac{\varepsilon}{2 T}\left(G_{\lambda_{2}}^{R}(\varepsilon / \hbar)-G_{\lambda_{2}}^{A}(\varepsilon / \hbar)\right) G_{\lambda_{1}}^{R}(\varepsilon / \hbar)\right\}
\end{array}
$$

where

$$
\mathbf{j}_{\lambda_{1} \lambda_{2}}(\mathbf{r})=\frac{i e \hbar}{2 m_{0}}\left\{\Phi_{\lambda_{1}}^{*}(\mathbf{r}) \nabla \Phi_{\lambda_{2}}(\mathbf{r})-\left(\nabla \boldsymbol{\Phi}_{\lambda_{\mathbf{1}}}^{*}(\mathbf{r})\right) \boldsymbol{\Phi}_{\lambda_{\mathbf{2}}}(\mathbf{r})\right\}-\frac{e^{2}}{m_{0} c} \mathbf{A}_{0} \Phi_{\lambda_{1}}^{*}(\mathbf{r}) \boldsymbol{\Phi}_{\lambda_{\mathbf{2}}}(\mathbf{r}) .
$$

Here $\Phi_{\lambda}(\mathbf{r})$ are the eigenfunction of the Hamiltonian and $\mathbf{A}_{0}$ is the vector potential of the applied static magnetic field. We consider the interband transitions and assume that the valence band states are occupied while the states in the conduction band are empty. Keeping only the resonant contribution in Eq.(75) we get

$$
\Pi_{\alpha \beta}^{R}\left(\mathbf{r}, \mathbf{r}^{\prime}, \omega\right)=\sum_{\lambda_{c} \lambda_{v}} j_{\lambda_{v} \lambda_{c}}^{\alpha}(\mathbf{r}) j_{\lambda_{c} \lambda_{v}}^{\beta}\left(\mathbf{r}^{\prime}\right) \int \frac{d \varepsilon}{2 \pi \hbar i} G_{\lambda_{c}}^{R}(\varepsilon / \hbar+\omega) G_{\lambda_{v}}^{A}(\varepsilon / \hbar),
$$

The states in the valence band we can consider as unchanged by the electron-phonon interaction (since we are interested only in the splitting phenomenon in the conduction band) and we get for the circularly polarized wave

$$
r_{+}=\frac{-i \Gamma\left(\hbar \omega-\varepsilon_{1}-\hbar \omega_{\mathrm{LO}}+\varepsilon_{v}\right)}{\left(\hbar \omega-\varepsilon_{+}+\varepsilon_{v}+i 0\right)\left(\hbar \omega-\varepsilon_{-}+\varepsilon_{v}+i 0\right) / \hbar+i \Gamma\left(\hbar \omega-\varepsilon_{1}-\hbar \omega_{\mathrm{LO}}+\varepsilon_{v}\right)},
$$

where we have introduced notation

$$
\Gamma=\frac{4 \pi}{\hbar \omega c \sqrt{\epsilon_{b}}} \sum_{\lambda_{c} \lambda_{v}} \int d \mathbf{r}^{\prime} d z j_{\lambda_{c} \lambda_{v}}^{x}\left(\mathbf{r}^{\prime}\right) j_{\lambda_{v} \lambda_{c}}^{x}(\mathbf{r})
$$

This quantity can be related to the recombination rate of the transition under consideration. Here we have taken into account that $\Pi_{x x}=\Pi_{y y}$. Since we are interested in the splitting phenomenon we assume that the resonance

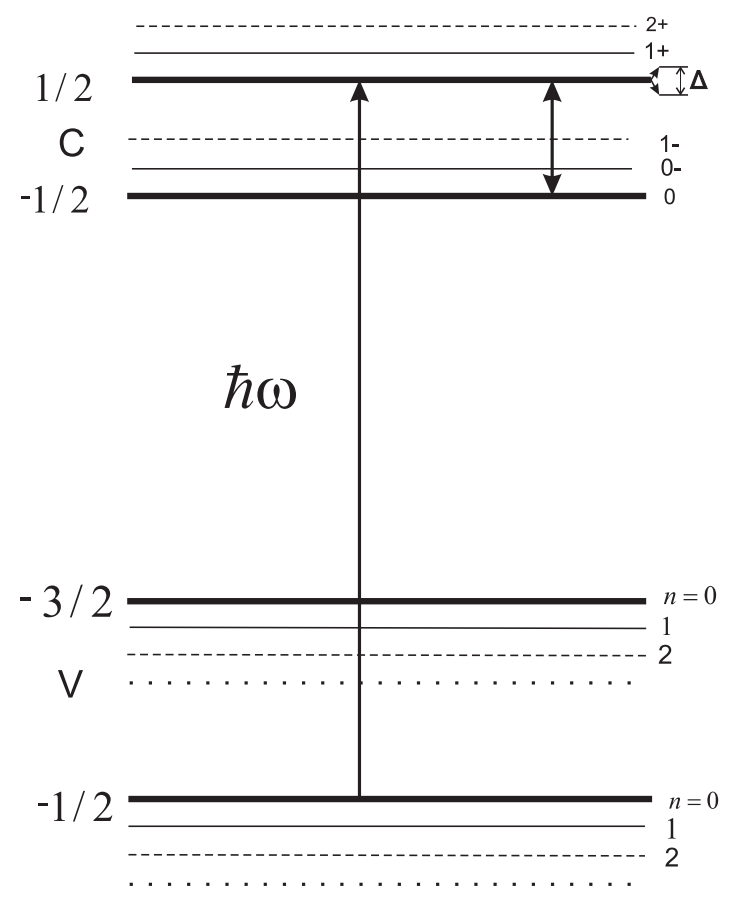

FIG. 3: Interband transitions.

light frequency is close to the transition from the ground state in the + group in the conduction band to the ground state in the valence band with $J_{z}=-1 / 2\left(\operatorname{see}\right.$ Fig[3] [24]. Therefore, in the following formulae we set $\varepsilon_{1}=\varepsilon_{c 1}=\varepsilon_{0}$, $\varepsilon_{2}=\varepsilon_{c 2}=\varepsilon_{0+}, \varepsilon_{c 2}-\varepsilon_{c 1}=\mu_{B} g_{c} B, \varepsilon_{v}=-E_{-1 / 2,0,1}^{(\mathrm{lh})}$ and $\Delta=\Delta_{0+, 0}$. 
The specification of the transition between the states described by Eq.(58) and

$$
\psi_{c 0+}=S \chi_{n_{c}}(z)\left(\varphi_{0}\left(x-x_{0 k_{y}}\right) s_{+}+u_{0} \varphi_{1}\left(x-x_{0 k_{y}}\right) s_{-}\right) \frac{e^{i k_{y} y}}{\sqrt{L_{y}}}
$$

allows us to express the recombination rate explicitly

$$
\Gamma=\frac{4 \pi}{\hbar \omega c \sqrt{\epsilon_{b}}} \frac{e^{2}\left|p_{c v}\right|^{2}}{6 m_{0}^{2}} \frac{1}{2 \pi l_{c}^{2}}, \quad p_{c v}=<S\left|p_{x}\right| X>
$$

Here we have assumed $\mid\left\langle\chi_{v 1}\left|\chi_{c 1}>\right|^{2}=1\right.$ for the overlapping of the transverse quantized wave function of the conduction and valence bands. Now we will introduce the following dimensionless variables: the deviation from the $\operatorname{SMPR}\left(\varepsilon_{c 2}-\varepsilon_{c 1}-\hbar \omega_{\mathrm{LO}}\right) / \Delta=\delta$, the optical frequency $2 \hbar\left(\omega-\omega_{0}\right) / \Delta=x\left[\omega_{0}=\left(\varepsilon_{c 2}-\varepsilon_{v}\right) / \hbar\right.$ being the interband resonance frequency], and the uncertainty in the level energy position $2 \hbar \Gamma / \Delta=\gamma$. Then we can write the power reflection coefficient $R=\left|r_{+}\right|^{2}$ as

$$
R=\frac{\gamma^{2}(x+2 \delta)^{2}}{\left(x+\delta-\sqrt{1+\delta^{2}}\right)^{2}\left(x+\delta+\sqrt{1+\delta^{2}}\right)^{2}+\gamma^{2}(x+2 \delta)^{2}} .
$$

If the dimensionless deviation $\delta \gg 1$ (i.e. the deviation from the phonon resonance condition is much bigger than the splitting) using $\sqrt{1+\delta^{2}} \simeq \delta$ we see that the single line structure is restored

$$
R=\frac{\gamma^{2}}{x^{2}+\gamma^{2}}
$$

In the case of exact electron phonon resonance that can be achieved by sweeping the magnetic field, $\delta=0$ and we get

$$
R=\frac{\gamma^{2} x^{2}}{\left(x^{2}-1\right)^{2}+\gamma^{2} x^{2}}
$$

In this case the power reflection coefficients reaches its maximal value under the optical resonant conditions. For linearly polarized incident wave this maximal value is $1 / 2$.

So far we have assumed that the energy uncertainty of the level under consideration is much smaller than the splitting $\Delta$, otherwise the level splitting can not be resolved. Indeed, we will have for the Green function instead of Eq.(15)

$$
G_{2}(\varepsilon)=\frac{1}{\varepsilon+i \hbar \Gamma_{2}-\varepsilon_{2}+\mu-(\Delta / 2)^{2} /\left(\varepsilon+i \hbar \Gamma_{1}-\varepsilon_{1}-\hbar \omega_{L O}+\mu\right)}
$$

provided we take this uncertainty into account. Here we have phenomenologically introduced $\Gamma_{2}$ and $\Gamma_{1}$ for the corresponding energy levels $\varepsilon_{2}$ and $\varepsilon_{1}$. It is seen from this expression that even for $\varepsilon-\varepsilon_{1}-\hbar \omega_{\mathrm{LO}}=0$ we can discard the second term in the denominator since $\Delta \ll \hbar \Gamma_{1}$ and the level does not split. The recombination rate can be estimated taking into account that $\left|p_{c v}\right|^{2} / 2 m_{0}$ is of the order of the Bohr energy, $\hbar \omega \sim E_{g} \sim 1.6 \mathrm{eV}$. Then it is seen that $\gamma=\hbar \Gamma / \Delta \ll 1$ as is assumed in Fig 4 .

Let us consider the case of equal $\Gamma_{1}=\Gamma_{2}$ widths of both levels. Then we can write for the reflection coefficient

$$
R=\frac{\gamma^{2}\left[(x+2 \delta)^{2}+\gamma_{e}^{2}\right]}{\left(x+\delta-\sqrt{1+\delta^{2}+\gamma_{e}^{2}}\right)^{2}\left(x+\delta+\sqrt{1+\delta^{2}+\gamma_{e}^{2}}\right)^{2}+4 \gamma_{e}^{2}(x+\delta)^{2}},
$$

where we introduce a dimensionless quantity proportional to the sum of level widths $\gamma_{e}=4 \hbar \Gamma_{1} / \Delta$ and neglect the level width due to recombination processes. Fig. 5 demonstrates how the increasing of the level widths smears the doublet structure of the reflection line. The symmetry of this doublet structure depends on the deviation from the spin electron-phonon resonance (Fig. 6).

\section{APPLICABILITY OF PERTURBATION THEORY}

In Sec II we considered only the simplest diagram for the self energy. Now we are going to discuss the validity of this approximation for the spin-phonon interaction. It is easily seen that each additional phonon line in the higher order diagrams can bring about additional resonant denominator, therefore we should consider the series of the most diverging sequence of diagrams. The situation is not unique and has been encountered earlier in the polaron problem in the three dimensional case and first such a consequence of diagrams has been considered by Pitaevskii [25]. 


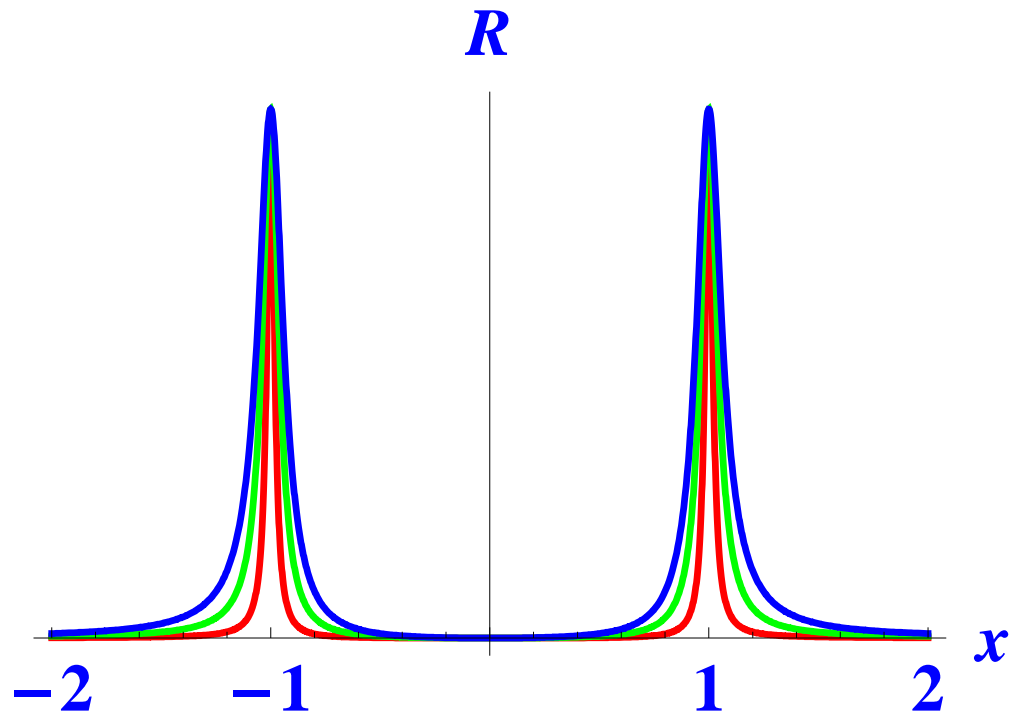

FIG. 4: Power reflection coefficient for SMPR as a function of optical frequency $x$ for $\gamma=0.04,0.09,0.14$. Here only the natural level width (due to recombination processes) is taken into account.

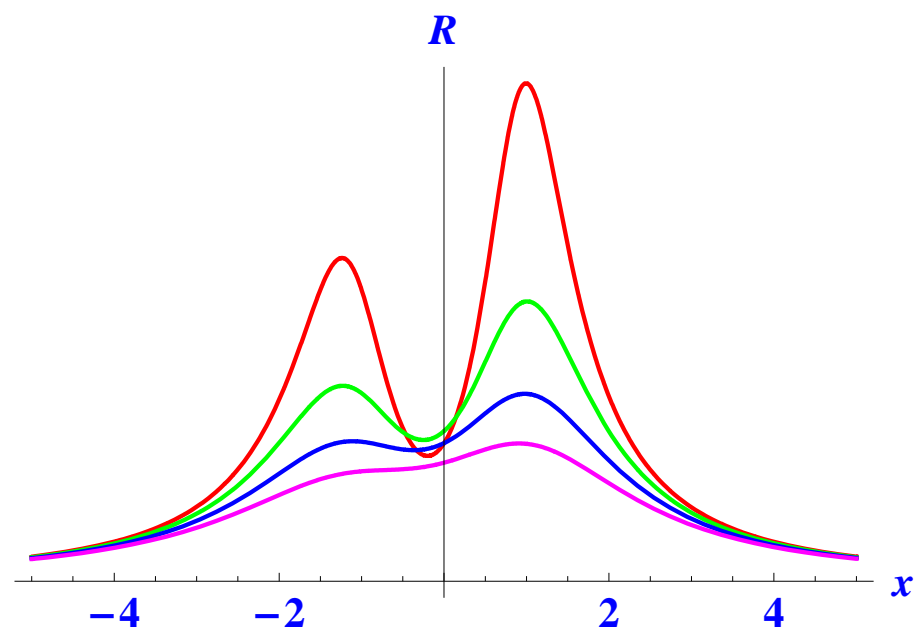

FIG. 5: Reflection coefficient as a function of optical frequency at $\delta=0.1$ for different level widths $\gamma_{e}=0.7,1,1.3,1.6$.

We consider two empty states 1 and 2 with energies $\varepsilon_{1,2} \varepsilon_{2}=\varepsilon_{1}+\hbar \omega_{\mathrm{LO}}$. Each state is unoccupied $\varepsilon_{1,2}>\mu$. Therefore we can write for the electron Green's function

$$
G\left(\varepsilon, \mathbf{r}_{1}, \mathbf{r}_{2}\right)=\sum_{\nu=1}^{2} \frac{\Psi_{\nu}\left(\mathbf{r}_{1}\right) \Psi^{*}\left(\mathbf{r}_{2}\right)}{\varepsilon-\left(\varepsilon_{\nu}-\mu\right)+i 0}
$$

The phonon Green function can be written as

$$
D\left(\omega, \mathbf{r}_{1}, \mathbf{r}_{2}\right)=\sum_{\alpha \mathbf{q}}\left|C_{\alpha, \mathbf{q}}\right|^{2}\left(\frac{e^{-i \mathbf{q}\left(\mathbf{r}_{1}-\mathbf{r}_{2}\right)}}{\omega-\hbar \omega_{\mathrm{LO}}+i 0}-\frac{e^{i \mathbf{q}\left(\mathbf{r}_{1}-\mathbf{r}_{2}\right)}}{\omega+\hbar \omega_{\mathrm{LO}}-i 0}\right),
$$

where $\left|C_{\alpha}\right|^{2} \rightarrow 2 \pi e^{2} \hbar \omega_{\mathrm{LO}} / q^{2} \epsilon^{*}$. We are to evaluate the Green function for the state 2 . Since we consider the empty electron states above the chemical potential the self energy diagrams will involve Green functions of the type

$$
\frac{1}{\varepsilon-\omega-\left(\varepsilon_{1,2}-\mu\right)+i 0} .
$$

These functions have the pole with respect to $\omega$ in the upper half-plane. Therefore we keep in the phonon Green's function only the part having the pole with respect to $\omega$ in the lower half-plane (otherwise the integration over $\omega$ 


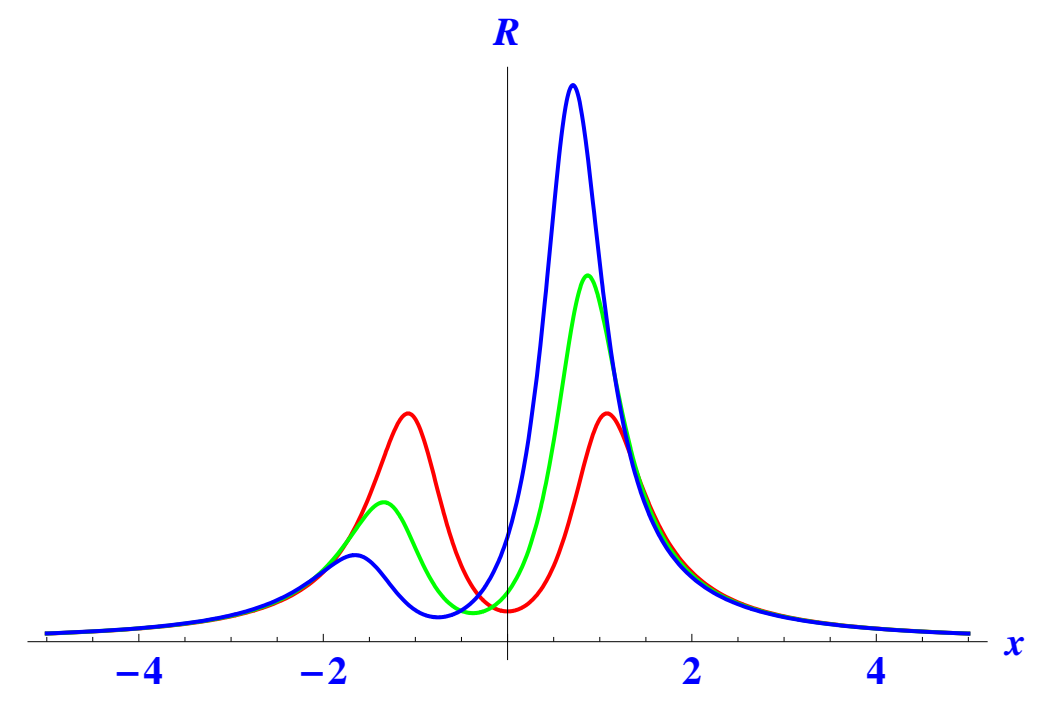

FIG. 6: Reflection coefficient for different deviations from the spin electron-phonon resonance $\delta=0,0.2,0.4$ at $\gamma_{e}=0.5$.

vanishes), i.e.

$$
\frac{1}{\omega-\omega_{\mathrm{LO}}+i 0} \text {. }
$$

The simplest electron self energy diagram (see diagram (a) in Fig.7) has a resonant denominator

$$
\Sigma_{2}(\varepsilon) \sim i \int \frac{d \omega}{2 \pi} G(\varepsilon-\omega) D(\omega)=\frac{1}{\varepsilon-\omega_{\mathrm{LO}}-\left(\varepsilon_{1}-\mu\right)+i 0},
$$

when $\varepsilon$ is in the vicinity of $\varepsilon_{2}=\varepsilon_{1}+\omega_{\mathrm{LO}}$. Diagrams with more resonances are of two types: the first type leads

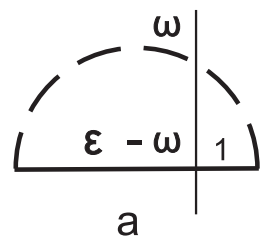

a
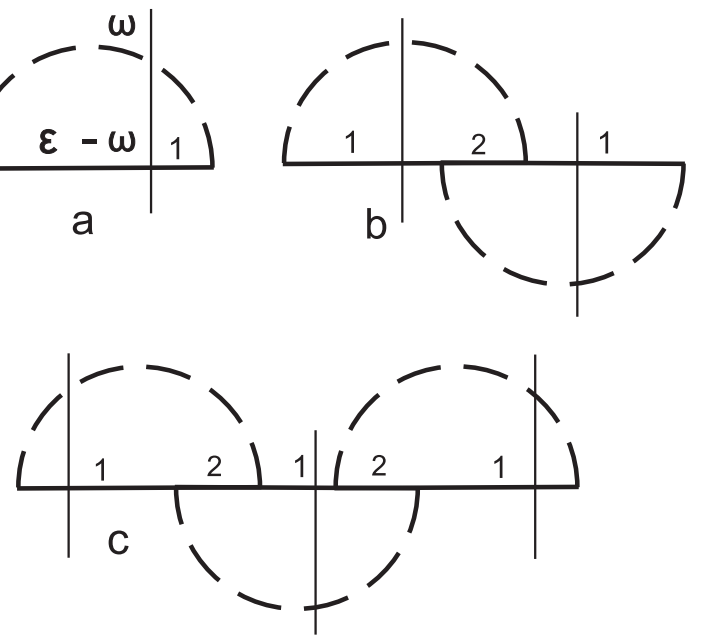

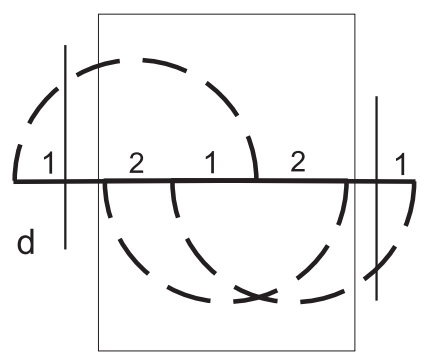

FIG. 7: Self energy diagrams. Resonant sections are shown by vertical lines

to corrections to the Green function (to the line 1 in the skeleton diagram (a) in Fig (7)) and they can be taken into account regarding the Green function as renormalized, the second type leads to the corrections to the electronphonon vertex. Since the corrections of the first type can be taken into account perturbatively (these diagrams do not involve resonant denominators) we will not consider them and concentrate on the diagrams of the second type. Several diagrams of the last type are presented in Fig:7 The diagrams $(b)$ and $(c)$ involve two and three resonant denominators, respectively. We can draw more complicated diagrams with two resonance denominators (similar to diagram (d) in Fig.7D), it is now seen that the diagrams of this type can be regarded as the diagram (b) with a block that does not involve resonant denominators, such a block we will call a compact block. Therefore, we can write the integral equation for the renormalized vertex (see Fig 8 ). In the Fig 9 we show that the compact block is the 
expansion with respect to electron-phonon coupling strength, therefore we write the integral equation keeping only the first term in this expansion

$$
\begin{array}{r}
\Gamma\left(\varepsilon-\omega, k_{y}-q_{y}, \varepsilon, k_{y}, q_{x}, q_{z}\right)=<1 k_{y}-q_{y}\left|e^{-i q_{x} x-i q_{z} z}\right| 2 k_{y}>+ \\
+i \int \frac{d \omega^{\prime} d \mathbf{q}^{\prime}}{(2 \pi)^{4}}<1 k_{y}-q_{y}\left|e^{i q_{x}^{\prime} x+i q_{z}^{\prime} z}\right| 2 k_{y}-q_{y}-q_{y}^{\prime}><2 k_{y}-q_{y}-q_{y}^{\prime}\left|e^{-i q_{x} x-i q_{z} z}\right| 1 k_{y}-q_{y}^{\prime}>\times \\
\times \frac{\left|C_{\mathbf{q}^{\prime}}\right|^{2} \Gamma\left(\varepsilon-\omega^{\prime}, k_{y}-q_{y}^{\prime}, \varepsilon, k_{y}, q_{x}^{\prime}, q_{z}^{\prime}\right)}{\left(\omega^{\prime}-\omega_{\mathrm{LO}}+i 0\right)\left(\varepsilon-\omega-\omega^{\prime}-\left(\varepsilon_{2}-\mu\right)+i 0\right)\left(\varepsilon-\omega^{\prime}-\left(\varepsilon_{1}-\mu\right)+i 0\right)} .
\end{array}
$$

Let us write this equation for the specific states $1=\{n=0, i=1\}$ and $2=\{n=0+, i=1\}$ ( $\operatorname{see}$ Sec 【II), i.e. we

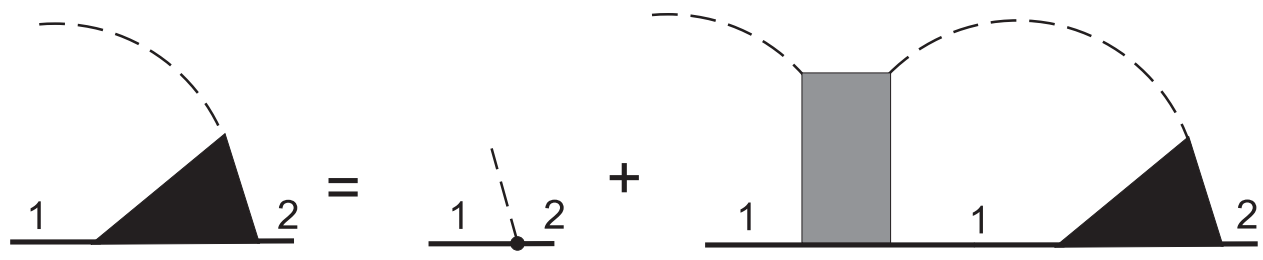

FIG. 8: Equation for the vertex
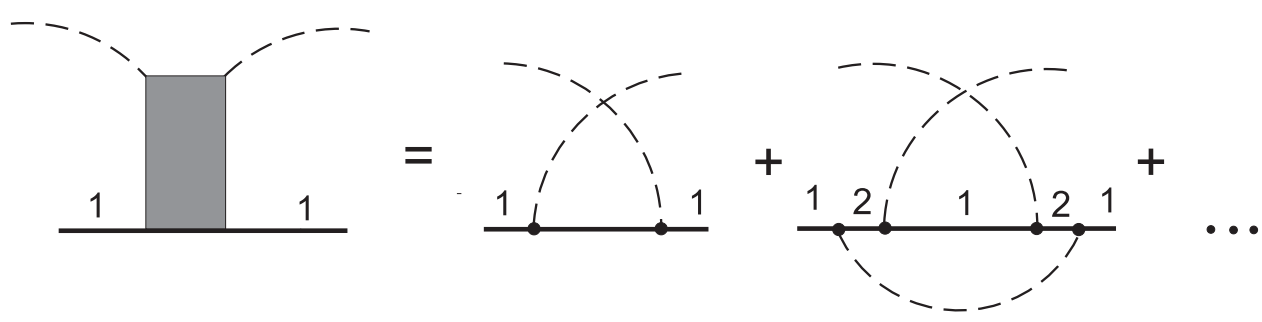

FIG. 9: Block expansion

consider the ground states with respect to orbital motion and to spatial confinement. In order to simplify the integral equation we introduce the function $A\left(q_{\perp}, \varepsilon, \omega\right)$ by relation

$$
\Gamma\left(\varepsilon-\omega, k_{y}-q_{y}, \varepsilon, k_{y}, q_{x}, q_{z}\right)=e^{i q_{x}\left(k_{y}-q_{y} / 2\right)} e^{-q_{\perp}^{2} / 4} \frac{q_{y}-i q_{x}}{\sqrt{2}}<1\left|e^{-i q_{z} z}\right| 1>A\left(q_{\perp}, \varepsilon, \omega\right),
$$

where $q_{\perp}=\sqrt{q_{x}^{2}+q_{y}^{2}}$ and wave vectors are dimensionless (the factor is the magnetic length). Then, using for the phase factors under the integral the relation

$$
e^{i\left(q_{y} q_{x}^{\prime}-q_{x} q_{y}^{\prime}\right)}=\sum_{n=-\infty}^{\infty} J_{2}\left(q_{\perp} q_{\perp}^{\prime}\right) e^{-i n\left(\varphi^{\prime}-\varphi\right)}
$$

we get

$$
\begin{gathered}
A(q, \varepsilon, \omega)=1+\frac{i}{2} \int \frac{d \omega^{\prime} p^{3} d p}{(2 \pi)^{3}} e^{-p^{2} / 2} \frac{J_{2}(q p) \phi(p) A\left(p, \varepsilon, \omega^{\prime}\right)}{\left(\omega^{\prime}-\omega_{\mathrm{LO}}+i 0\right)\left(\varepsilon-\omega-\omega^{\prime}-\left(\varepsilon_{2}-\mu\right)+i 0\right)\left(\varepsilon-\omega^{\prime}-\left(\varepsilon_{1}-\mu\right)+i 0\right)} . \\
\phi(p)=\frac{u_{0}^{2}}{l_{c}} \int d q_{z}\left|C_{p, q_{z}}\right|^{2}|<1| e^{i q_{z} z / l_{c}}|1>|^{2} .
\end{gathered}
$$

Now we suppose that the function $A(q, \varepsilon, \omega)$ has no poles with respect to $\omega$ in the lower half complex plane and consider the case when the magnetic length is much bigger than the quantum well width. The last assumption leads to $\phi(p)=\pi / p l_{c}$ and we can rewrite the integral equation for $A\left(q, \varepsilon, \omega_{\mathrm{LO}}\right) \equiv A(q, \varepsilon)$ as the Fredholm equation

$$
A(q, \varepsilon)=1+\lambda \int_{0}^{\infty} d p p^{2} e^{-p^{2} / 2} J_{2}(q p) A(p, \varepsilon),
$$

where parameter $\lambda$ includes the resonant denominator

$$
\lambda=-\frac{1}{8} \frac{u_{0}^{2}\left(e^{2} / \varepsilon^{*} l_{c}\right)}{\varepsilon-\left(\varepsilon_{1}+\hbar \omega_{\mathrm{LO}}-\mu\right)+i 0} .
$$


In reality the uncertainty of the level $i \hbar / \tau$ enter the last equation instead of $i 0$. Let us evaluate the minimum of $\tau$ when we remain in the framework of perturbation theory and it is then sufficient to consider only the skeleton diagram for the self energy. With $\hbar \omega_{\mathrm{LO}}=0.02 \mathrm{eV}, B=3 \mathrm{~T}, m_{c}=0.1 m_{0}, \alpha_{R}=10^{-9} \mathrm{eV} \cdot \mathrm{cm}, \alpha=0.39$ we get that the perturbation scheme is valid for the relaxation times shorter than $\tau_{0}=5 \cdot 10^{-10}$ sec. On the other hand, to resolve the splitting the level uncertainty must be smaller than the level splitting $\Delta \simeq 5 \cdot 10^{-4} \mathrm{eV}$. This requires the times bigger than $10^{-12}$ sec. Therefore, there exists a region of relaxation times $\sim 10^{-11}$ sec., where the perturbation theory is valid and the splitting phenomena is discernable.

Here we wish to note that in the ordinary situation of the magnetophonon phenomena one meets the opposite case of big $\lambda$ parameter in Eq.(97) and the integral equation should be solved. Therefore, we suppose that the theories taking into account only the one phonon processes described by the skeleton self energy diagram can not be considered as reliable.

\section{CONCLUSIVE REMARKS}

We have considered optical manifestation of SMPR in semimagnetic semiconductors. Due to the electron-phonon coupling the resonant reflection and transmission line representing the interband transitions is split into two lines. The distance between the lines is determined by the strength of electron-phonon coupling.

We should, however, indicate that some points have not been taken into account in our calculation. Among them the most important is the natural width of the phonon levels. For the optical phonons at low temperatures it is determined by decay of an optical phonon into two acoustic ones.

The natural width of the electron lines is also important. It may be determined by collisions of electrons with acoustic phonons and with defects of the lattice, as well as by recombination. These effects result in widening of the lines that has been briefly discussed. Under the conditions where these effects are strong, the lines may overlap as has been indicated above.

So far we have considered a situation where the equilibrium concentration of the carriers is so low that they do not influence the light absorption. One can conceive, however, another case of interest where, for instance, in equilibrium electrons (provided by donors outside the well) fill the conduction band up to the Fermi level. In such a case transitions between the valence band and the states of the conduction band above the Fermi level are allowed. The oscillator strength for these transitions may be bigger than for those treated in the present paper. One can expect that the width of the electron level in the conduction band should be rather small as the electrons can emit acoustic phonons with the energies not bigger than the spacing between the level they occupy and the Fermi level. However, one can expect that the width of the level in the valence band may be much bigger. Indeed, the holes can emit phonons with comparatively large energies as the spacing between their level and the top of the valence band can be rather large.

Experimental observation of SMPR can provide information about the electron-phonon interaction. Its investigation can also provide important information concerning various contributions into spin-orbit interaction as well as the strength of the exchange interaction.

\section{Acknowledgments}

The authors are grateful to Yu. G. Kusrayev for an interesting discussion where the topic of the present investigation emerged.

[1] V. L. Gurevich, Yu. A. Firsov, Zh. Eksp. Teor. Fiz. 40, 199, 1961 [Sov. Phys.-JETP 13, 137]

[2] Yu. A. Firsov, V. L. Gurevich, R. V. Parfeniev, I. M. Tsidil'kovskii, in Landau Level Spectroscopy edited by G. Landwehr and E. I. Rashba (Elsevier, Amsterdam, 1991)

[3] Pavlov S. T., Yu. A. Firsov, Fiz. Tverd. Tela 7, 2634, 1965[Sov. Phys.-Solid State 7, 2131,1966], Zh. Eksp. Teor. Fiz. 49, 1664, 1965 [Sov. Phys.-JETP 22, 1137,1966], Fiz. Tverd. Tela 9, 1780, 1967 [Sov. Phys.-Solid State 9, 1394]

[4] S. T. Pavlov and Yu. A. Firsov, Fiz. Tverd. Tela 9, 1780 (1967) [Sov. Phys. - Solid State 9, 1394 (1967)].

[5] I. M. Tsidilkovskii, M. M. Aksel'rod, and S. I. Uritskii, Phys. Status Solidi 12, 667 (1965).

[6] W. Zawadzki, G. Bauer, and H. Kahlert, Phys. Rev. Lett. 35, 1098 (1975).

[7] Komarov A. V., Ryabchenko S. M., Terletskii O. V., Zheru I. I., Ivanchuk R. D., Zh. Eksp. Teor. Fiz. 73, 608,1977 [Sov. Phys.-JETP 46, 318]

[8] Gaj J. L., Galazka R. R., Nawrocki M., Solid. State Commun., 25, 193, 1978

[9] Bartholomew D. U., Furdyna J. K., Ramdas A. K., Phys. Rev. B 34, 6943, 1986

[10] A. K. Bhattacharjee, Fishman G., Coqblin B., Physica 117B and 118B, 449, 1983

[11] B. E. Larsen, K. C. Hass, H. Ehrenreich, A. E. Carlsson, Phys. Rev. B 37, 4137, 1988

[12] Bhattacharjee A. K., Phys. Rev. B, 41, 5696, 1990

[13] L. I. Korovin, S. T. Pavlov, Zh. Eksp. Teor. Fiz. 53, 1708, 1967 [Sov. Phys.-JETP 26, 979, 1968]

[14] S. Das Sarma, A. Madhukar, Phys. Rev. B, 22, 2823, 1980 
[15] I. G. Lang, L. I. Korovin, S. T. Pavlov, Fiz. Tverd. Tela, 47,1704, 2005)[Phys.-Solid State,47, 2005]; Fiz. Tverd. Tela, 48,1693, 2006 [Phys.-Solid State,48, 2006]

[16] J. A. Gaj, R. Planel, G. Fishman, Solid State Commun., 29, 435, 1979

[17] N. Mori, T. Ando, Phys. Rev. B, 40, 6175, 1989

[18] H. Fröhlich, Adv. Phys., 3,2, 325, 1954

[19] Bychkov Yu. L., Rashba E. I., Pisma Zh. Eksp. Teor. Fiz., 39, 66, 1984 [Sov. Phys.-JETP 39, 78, 1984]

[20] G. Dresselhaus, Phys. Rev., 100, 580, 1955

[21] Gradshteyn I. S., I. M. Rhyzhik, Tables of integrals, series and products (Academic Press, New York, 1965)

[22] Bir G. L., Pikus G. E., Symmetry and Strain-Induced Effects in Semiconductors, (Wiley, New York, 1974)

[23] R. Winkler, Phys. Rev. B, 62, 4245, 2000

[24] Note that the spin-orbit interaction can allow optical transitions from this group to the valence band top states with $J_{z}=-3 / 2$ but the intensity of these transitions is proportional to the small interaction constant $u_{0}$.

[25] L. P. Pitaevskii, Zh. Eksp. Teor. Fiz. 36, 1168, 1959 [Sov. Phys. -JETP 19, 1959] 